\title{
Efficacy of endoscopic vascular staplers for splenic hilar control during laparoscopic splenectomy
}

\author{
Barış Özcan, ${ }^{1} \oplus$ Metin Çevener, ${ }^{2} \oplus$ Burak Deveci, ${ }^{3} \odot$ Ihssan Karadoğan, ${ }^{3} \odot$ Okan Erdoğan ${ }^{1}$ \\ 'Department of General Surgery, Medstar Antalya Hospital, Antalya, Turkey \\ ${ }^{2}$ Department of Radiology, Medstar Antalya Hospital, Antalya, Turkey \\ ${ }^{3}$ Department of Hematology, Medstar Antalya Hospital, Antalya, Turkey
}

\begin{abstract}
Introduction: This study aimed to assess the results and effectiveness of using endoscopic vascular staplers for splenic hilar control in 36 consecutive cases who underwent elective laparoscopic splenectomy due to hematologic problems.

Materials and Methods: Thirty-six consecutive patients who underwent laparoscopic splenectomy (LS) due to hematologic diseases over a 3-year period between January 2012-May 2015 were included in this study. LS was carried out with the standard 4-port method. All hilar vessels were divided by using an endoscopic stapler loaded with vascular cartridges. Patient demographic data, hematologic diagnosis, size of the spleen, duration of operation, length of hospital stay, post-operative complications, postoperative analgesic requirement were retrospectively extracted from patient files.

Results: A total of 36 consecutive patients were treated in a single center and were operated by two surgeons. There was no conversion to laparotomy or reoperation in the series. One patient developed acute portal vein thrombosis that was medically treated in the early post-operative period. Another patient experienced pneumothorax due to a diaphragmatic injury that was repaired during surgery. The average spleen size was identified as $14.5 \mathrm{~cm}$. An accessory spleen was detected in 5 patients. The mean operative time was 97 minutes, and the mean length of hospital stay was 2.3 days.

Conclusion: We believe that using an endoscopic vascular stapler for ligation of hilar vascular structures during elective laparoscopic splenectomy by experienced surgeons is efficient despite the cost disadvantage.
\end{abstract}

Keywords: Endoscopic vascular stapler; laparoscopic splenectomy; splenic hilum.

\section{Introduction}

Following its introduction by Delaitre et al. ${ }^{[1]}$ in 1991, laparoscopic splenectomy (LS) has gained wide acceptance and has become the treatment of choice for elective splenectomy. ${ }^{[2]}$ LS technique has improved in time parallel with technological developments. The dissection and control of splenic vascular structures was generally achieved by endoscopic vascular staplers for many years. With introduction of new vessel sealing devices such as 
Harmonic scalpel (Ethicon Endo-Surgery, Cincinnati, OH) and Ligasure (Valley Lab, Boulder CO, USA), it has been reported that these tools are both safe and reliable in the dissection and sealing of splenic pedicles. ${ }^{[3]}$ Currently, LS is being successfully performed for the treatment of both benign and malignant hematologic diseases as well as other primary splenic disorders. ${ }^{[4-6]}$

In this study, we aimed to present a case series of 36 consecutive patients with hematologic diseases who underwent elective LS by only using an endoscopic vascular stapler for splenic hilar control, and to report our singlecenter results.

\section{Materials and Methods}

36 consecutive patients who underwent LS due to hematologic diseases over a 3-year period between January 2012-May 2015 were included in this study. Patient demographic data, hematologic diagnosis, size of the spleen, duration of operation, length of hospital stay, volume of post-operative drainage, postoperative analgesic requirement were retrospectively extracted from patient files.

This study was conducted in accordance with the ethical standards set out in the Helsinki Declaration.

\section{Indications}

The patients who were referred to the general surgery department from hematology department had benign or malignant hematologic diseases. Twenty-nine patients (80\%) underwent therapeutic LS for idiopathic thrombocytopenic purpura (ITP). Three patients diagnosed with myelodysplastic syndrome (MDS) and one patient with beta-thalassemia underwent LS for both diagnostic and therapeutic purposes. Three patients with a presumptive diagnosis of lymphoma underwent diagnostic LS (Table 1).

\section{Pre-operative Preparation}

Preoperative abdominal ultrasound was performed in all patients to determine the size of the spleen. All patients

\begin{tabular}{lc}
\hline Table 1. Patient indications for laparoscopic \\
splenectomy \\
Idiopathic thrombocytopenic purpura & 29 \\
Myelodysplastic syndrome & 3 \\
Beta-Thalassemia & 1 \\
Lymphoma & 3
\end{tabular}

were vaccinated 2 weeks before or 2 weeks after surgery against Streptococcus pneumoniae, Neisseria meningitidis and Haemophilus influenzae.

Preoperative platelet count was measured in all patients with a complete blood count. Patients with a platelet count below $50 \times 10^{9} / \mathrm{L}$ were preoperatively hospitalized to the hematology clinic and received prednisolone and intravenous immunoglobulin. In the meantime, anesthesia evaluation was completed and supply of fresh frozen plasma, red blood cell and platelet concentrate (apheresis) were prepared. The operation was performed after the platelet levels were increased up to at least $100 \times 10^{9} / \mathrm{L}$. 3 ITP patients were resistant to corticosteroid and IVIG treatment or pre-operative platelet replacement, thus they had to be taken to surgery with platelet levels below $50 \times 10^{9} / \mathrm{L}$. Informed consent taken from all patients. One of these 3 patients was a full-term pregnant with ITP.

\section{Surgical Procedures and Techniques}

All surgical operations were performed by two surgeons with advanced laparoscopic surgery experience who have previously performed laparoscopic splenectomies by using different techniques in other hospitals.

All patients were operated under general anesthesia. Nasogastric decompression and urethral catheterization were routinely used. A single dose of 3rd generation cephalosporin was administered intravenously 30 minutes prior to surgery for antibiotic prophylaxis. All operations were performed in the right lateral decubitus position (Fig. 1).

LS was carried out with the standard 4-port technique. A 10-mm telescope port was inserted at the mid-clavicular line approximately 4-6 cm below the costal margin with the open technique. Pneumoperitoneum was achieved by

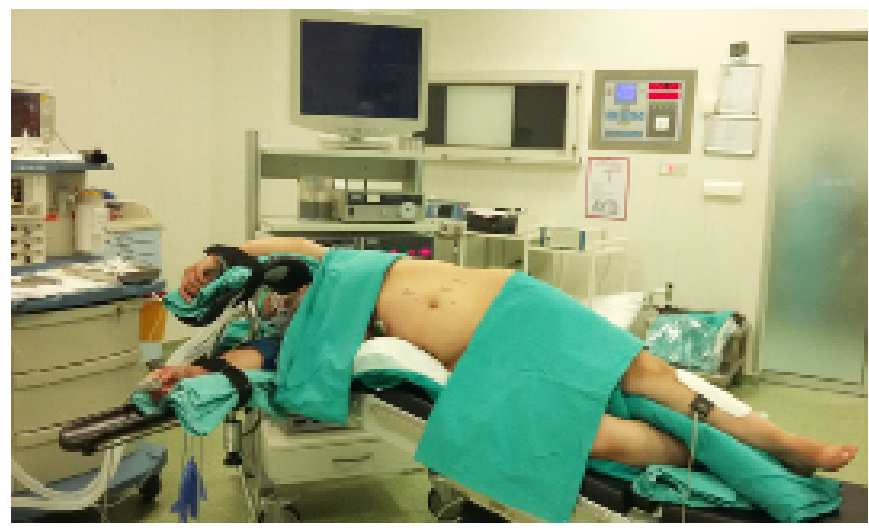

Figure 1. Right lateral decubitus position. 
$\mathrm{CO}_{2}$ insufflation. A $15-\mathrm{mm}$ port was placed at the intersection point of the anterior axillary line and the sub-costal area in the left flank and another 5-mm port was inserted more laterally, all under direct vision (Fig. 2). One more 5 $\mathrm{mm}$ port was placed in the midline in the epigastric area, distal to the xiphoid. The ports had to be placed more medially or through the lower abdominal quadrant in some patients according to the size of the spleen. Monitors were placed in the upper left corner of the operating table.

The splenocolic and splenorenal ligaments were divided upwards beginning from the lower pole by using monopolar, bipolar diathermy and Ligasure (Valley Lab, Boulder CO, USA). The gastrosplenic ligament was transected and the short gastric vessels were divided by using Ligasure. Then, the spleen was fully mobilized by dissecting the splenophrenic ligament. In all patients, the pancreatic tail was clearly distinguished from the spleen hilus. Sufficient space was created between the spleen and the pancreas to allow placement of endoscopic vascular staples. The splenic hilum was visualized and was transected with an endoscopic stapler loaded with $30-45-60 \mathrm{~mm}$ vascular cartridges according to hilar length. Multiple firings

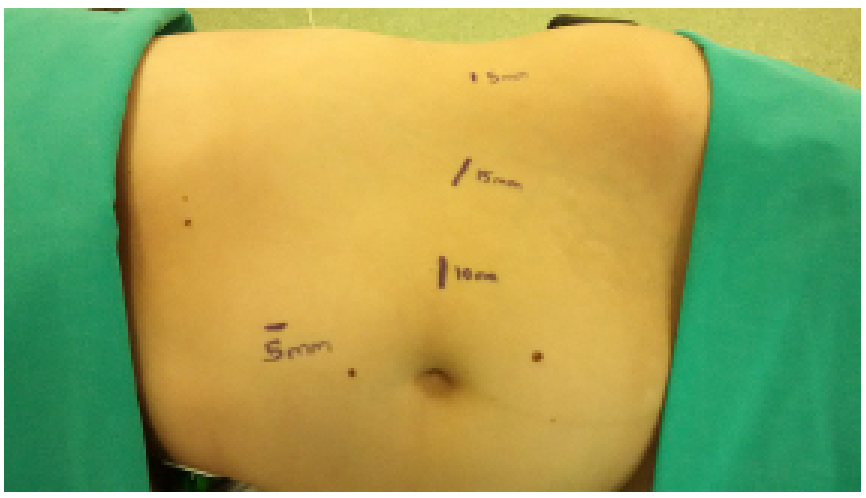

Figure 2. Trocar size and sites.

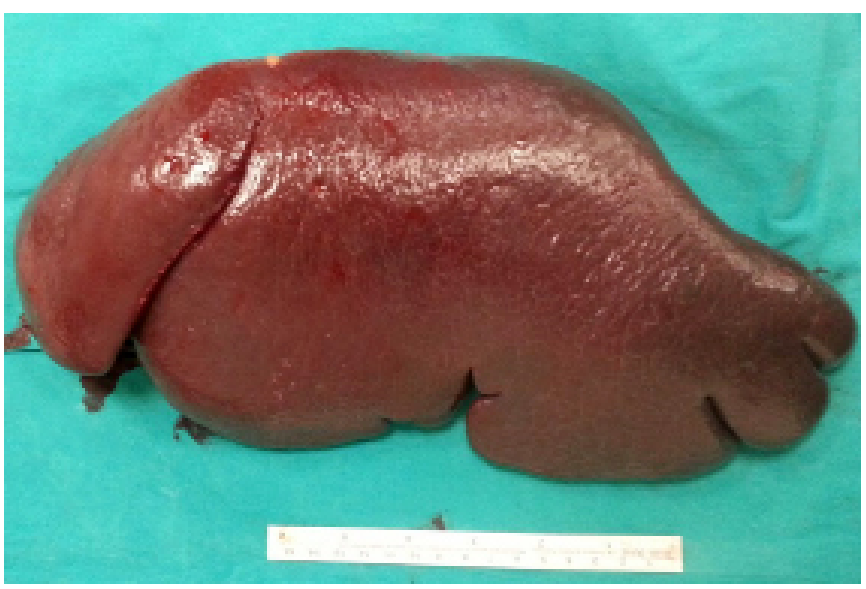

Figure 3. A large spleen specimen following laparoscopic splenectomy. were required in 21 patients. Once the spleen was fully released and de-vascularized, it was extracted from the 15 $\mathrm{mm}$ port in an endo-bag (Endo Catch, Auto Suture Covidien, Mansfield, USA) with piecemeal extraction. In case of an extremely large spleen or a necessity to remove the spleen intact, the spleen was extracted through a limited Pfannenstiel incision performed on the left lower quadrant (Fig. 3). Hemostasis was confirmed in the stapler line and short gastric vessels. A surgical drain was placed in all cases.

\section{Post-operative Care}

The nasogastric tube was removed at the end of the operation and liquid diet was started 6-8 hours after surgery. Intravenous tramadol infusion was used for post-operative analgesia.

Complete blood count was obtained in the first post-operative morning in each patient. Once the patients were mobilized and their drainage amounts were evaluated, the drains were withdrawn either on the first or second postoperative day if the drainage was less than $50 \mathrm{~mL}$. After being discharged, the patients were evaluated weekly in general surgery and hematology outpatient clinics. If their platelet counts exceeded $300 \times 10^{9} / \mathrm{L}$ they were prescribed aspirin or low molecular heparin for thrombotic events.

\section{Statistical Analyses}

The data recorded in the Microsoft Excel table were used in the analyses. The descriptive statistics were given as number and percentage for categorical variables and mean value, standard deviation, and minimum and maximum values for numerical variables.

\section{Results}

Thirty-six consecutive patients who underwent laparoscopic splenectomy due to hematologic diseases over a 3-year period between January 2012-May 2015 were included in this study. Twelve patients were male. The mean age was $44( \pm 15)$ years, length of hospital stay was 2.3 days, and follow-up time was 23 months. The average spleen size according to ultrasound results was found to be $14.5 \mathrm{~cm}$, and the maximum craniocaudal length was measured as $26 \mathrm{~cm}$ in a patient with ITP (Table 2).

All operations were completed by laparoscopy without the need for conversion to laparotomy. There was no postoperative mortality. The mean duration of operation was 
Table 2. Patient and operative-postoperative data

No. of patients (male/female)

Mean age, year (range)

$36(12 / 24)$

Average spleen diameter, $\mathrm{cm}$ (range)

$39( \pm 15)$

Average operating time, min (range)

Average hospital stay, d (range)

Morbidity (\%)

$14.5(8-26)$

$97(45-150)$

$2.3(1-5)$

5.5

Accessory spleens (\%)

97 minutes (45-150 minutes). Based on preoperative abdominal ultrasound results, the average spleen size was identified as $14.5 \mathrm{~cm}$, with the minimum and maximum craniocaudal length measuring $8 \mathrm{~cm}$ and $26 \mathrm{~cm}$, respectively. An accessory spleen was detected and removed in 5 patients (13.9\%) (Table 2).

Uncontrollable bleeding requiring re-operation or blood transfusion was not encountered in any patient. The mean post-operative drainage volume was $75 \mathrm{cc}$ (25-150 cc). One patient was re-hospitalized due to severe abdominal pain two days after discharge. An acute portal vein thrombosis was detected that was treated with heparin therapy. The patient was discharged at the $10^{\text {th }}$ day of re-hospitalization after complaints regressed and recanalization of portal flow was confirmed. In another patient, a minor left diaphragmatic injury was identified almost at the end of the operation, which was sutured primarily during laparoscopy along with left tube thoracostomy. The patient had no problems during follow-ups, and was discharged on the $5^{\text {th }}$ postoperative day.

There was no pancreatic tail injuries in any patient intraoperatively. In conclusion, no subdiaphragmatic abscess or pancreatic fistula developed.

The platelet count could only be elevated up to $23 \times 10^{9} / \mathrm{L}$ despite IVIG and replacement therapy before $\mathrm{C}$-section in a full-term pregnant patient with ITP. First, the C-section was performed via a Pfannenstiel incision and the incision was temporarily closed. Then, a standard LS procedure was performed and the $\mathrm{C}$-section incision was re-opened to extract the spleen. There were no complications during the intra-operative and post-operative periods.

An endoscopic stapler loaded with 60-mm vascular cartridge was used in most of our patients and hilar resection was completed with a single firing. Serial firings were required in some patients according to the size of the spleen and that of the splenic hilum. A $10-\mathrm{mm}$ or $15-\mathrm{mm}$ endo-

bag (Endo Catch, Auto Suture Covidien, Mansfield, USA) was used for splenic extraction. The spleen was extracted through a limited Pfannenstiel incision at the left lower quadrant without using an endo-bag (Endo Catch, Auto Suture Covidien, Mansfield, USA) in a pregnant patient whose $\mathrm{C}$-section procedure was carried out concurrently with LS, in 6 patients with massive splenomegaly whose spleen would not fit in an endo-bag (Endo Catch, Auto Suture Covidien, Mansfield, USA), and in two patients who have been previously diagnosed with malignancy.

Post-operative analgesia was provided by iv infusion of $200 \mathrm{mg}$ /day tramadol in patients with standard LS where the spleen was extracted by using an endobag without any additional incision. However, patients who required a Pfannenstiel incision experienced more pain with an increase in daily tramadol requirement to $400 \mathrm{mg}$.

\section{Discussion}

Laparoscopic splenectomy (LS) is the treatment of choice for both benign and malignant diseases of the spleen. ${ }^{[2,4-6]}$ The literature data suggest that LS has several advantages over open surgical procedures. ${ }^{[7-9]}$ We have performed LS to all patients who were referred to our clinic from hematology clinics for the diagnosis and treatment of benign or malignant hematologic diseases, unless there was a contraindication for laparoscopic surgery.

Various alternative methods and equipment are being used during LS procedure, with reports on their safety and reliability. ${ }^{[3,10-12]}$ Nevertheless, although vessel sealing devices such as Harmonic scalpel and Ligasure are considered to be efficient in vascular structures up to $7 \mathrm{~mm}$, the hemostatic reliability of these devices in larger diameter vessels or in case of bleeding tendency is controversial. ${ }^{[13]}$ It is also known that splenomegaly is associated with proliferation of splenic vascular structures and increased vessel diameters.

The surgeons who participated in this study have not used standard splenic hilar transection methods during their previous LS operations and have experience with different devices such as endoscopic vascular staplers, metal or hem-o-lock clips, Harmonic scalpel and Ligasure. It is the authors' opinion that performing LS by using clips or vessel sealing devices requires more detailed splenic hilar and vascular dissection that result in extra-time and hemorrhage. Indeed, the mean duration of operation was identified as 97 minutes in this case series, which can be regarded as quite short as compared to other case series 
in the literature. ${ }^{[3,10,12,14]}$ This decrease in operating time is attributed to performing splenic hilar resection, which is the most important and difficult stage of the operation, en bloc by using staplers.

Miles et al. ${ }^{[15]}$ reported 28 consecutive patients in whom they used endoscopic vascular staplers for splenic isolation during LS. They stated that all operations were completed without any serious complications and that using an endoscopic vascular stapler was a reliable method. Uranüs S. ${ }^{[16]}$ reported using endoscopic vascular staplers for hilar dissection in 89 patients who underwent laparoscopic total splenectomy, within their cohort of laparoscopic total or partial splenectomy over 14-years. They stated that performing hilar resection with endoscopic vascular staplers and $60 \mathrm{~mm}$ cartridges was quite faster, safer and more effective as compared to using hem-olock clips. In the case series of 107 patients undergoing LS, Vecchio et al. ${ }^{[17]}$ used endoscopic vascular staplers in all but 3 patients requiring conversion to laparotomy due to problems during splenic hilar resection. The authors stated that using endoscopic vascular staplers for splenic hilar transection during LS was a reliable and effective method. Our study also supported the efficiency and reliability of endoscopic vascular stapler use with a low complication rate and without the requirement to conversion to laparotomy.

The most frequent complications encountered during LS include sub-diaphragmatic abscess (3\%), pneumonia or atelectasis (2\%) and hemorrhage. ${ }^{[2,5,9]}$ Although pancreatic fistula and portal vein thrombosis (PVT) are listed as rare complications, PVT was detected in one patient in our study that was treated medically. In another patient, an iatrogenic diaphragmatic perforation occurred that was treated with laparoscopic repair and chest tube drainage during LS.

Intraoperative bleeding is the most important cause of conversion in LS procedures, while post-operative bleeding is the leading cause of re-operation and mortality. ${ }^{[2,5]}$ In our study, there was no significant intraoperative or postoperative bleeding. In other words, none of the study patients developed bleeding requiring conversion or reoperation. We believe that the use of endoscopic staplers significantly contributed to these results. It should be emphasized that the spleen must be fully separated from its attachments in order to be placed directly to the splenic hilum and that all hilar structures should be included between the jaws of the vascular cartridges.
There are studies indicating that the size of the spleen affects both the duration of surgery and complication rates. ${ }^{[18]}$ Patel et al. reported that the morbidity rate increases when the spleen size is over $15 \mathrm{~cm}$. In our study, the mean spleen size was determined as $14.5 \mathrm{~cm}$, and 18 patients had a spleen size over $15 \mathrm{~cm}$. In our case series, LS was successfully applied to a patient with splenomegaly with a maximum craniocaudal length of $26 \mathrm{~cm}$.

The incidence of accessory spleen in the general population is reported as $10 \%$, but this ratio increases up to $30 \%$ in hematologic diseases. ${ }^{[19]}$ The incidence of accessory spleen in LS ranges from $11-21 \% .^{[5,6]}$ Compatible with the literature, an accessory spleen was detected in 5 patients (13.9\%) in our study.

In our study, the mean length of hospital stay was determined as 2.3 days that seems to be shorter than many series in the literature. ${ }^{[3,9,11]}$ We believe that this is related to the low complication rates and to extracting the spleen through the port sites in most cases without performing an additional incision. Length of hospital stay is mainly related to analgesic requirements and the presence of complications. In our study, we determined that analgesic requirements of the patients who underwent limited Pfannenstiel incision was twice as high as compared to other patients.

\section{Conclusion}

We believe that using an endoscopic vascular stapler for ligation of hilar vascular structures during elective laparoscopic splenectomy by experienced surgeons is efficient despite the cost disadvantage.

\section{Disclosures}

Ethichs Committee Approval: Authors declared that the research was conducted according to the principles of the World Medical Association Declaration of Helsinki "Ethical Principles for Medical Research Involving Human Subjects" (amended in October 2013).

Informed Consent: Informed consent was not received due to the retrospective nature of the study.

Financial Disclosure: The authors declared that this study has received no financial support

Peer-review: Externally peer-reviewed.

Conflict of Interest: None declared. 


\section{References}

1. Delaitre B, Maignien B. Splenectomy by the laparoscopic approach. Report of a case [Article in French]. Presse Med $1991 ; 20: 2263$.

2. Feldman LS. Laparoscopic splenectomy: standardized approach. World J Surg 2011;35:1487-95. [CrossRef]

3. Gelmini R, Romano F, Quaranta N, Caprotti R, Tazzioli G, Colombo $\mathrm{G}$, et al. Sutureless and stapleless laparoscopic splenectomy using radiofrequency: LigaSure device. Surg Endosc 2006;20:991-4. [CrossRef]

4. Silecchia G, Boru CE, Fantini A, Raparelli L, Greco F, Rizzello $M$, et al. Laparoscopic splenectomy in the management of benign and malignant hematologic diseases. J Soc Laparosc Surg 2006;10:199-205.

5. Rosen M, Brody F, Walsh RM, Tarnoff M, Malm J, Ponsky J. Outcome of laparoscopic splenectomy based on hematologic indication. Surg Endosc 2002;16:272-9. [CrossRef]

6. Delaitre B, Champault G, Barrat C, Gossot D, Bresler L, Meyer C, et al. Laparoscopic splenectomy for hematologic diseases. Study of 275 cases. French Society of Laparoscopic Surgery [Article in French]. Ann Chir 2000;125:522-9. [CrossRef]

7. Winslow ER, Brunt LM. Perioperative outcomes of laparoscopic versus open splenectomy: a meta-analysis with an emphasis on complications. Surgery 2003;134:647-55. [CrossRef]

8. Musallam KM, Khalife M, Sfeir PM, Faraj W, Safadi B, Abi Saad GS, et al. Postoperative outcomes after laparoscopic splenectomy compared with open splenectomy. Ann Surg 2013;257:1116-23.

9. Pomp A, Gagner M, Salky B, Caraccio A, Nahouraii R, Reiner $M$, et al. Laparoscopic splenectomy: a selected retrospective review. Surg Laparosc Endosc Percutan Tech 2005;15:13943. [CrossRef]

10. Aydin C, Kayaalp C, Olmez A, Tatli F, Kirimlioglu V. Laparo- scopic splenectomy with a vessel sealing device. Minim Invasive Ther Allied Technol 2008;17:308-12. [CrossRef]

11. Canda AE, Ozsoy Y, Yuksel S. Laparoscopic splenectomy using LigaSure in benign hematologic diseases. Surg Laparosc Endosc Percutan Tech 2009;19:69-71. [CrossRef]

12. Shabahang $H$, Maddah G, Tavassoli A, Jangjoo A, Alvandipour $M$, Abdollahi $A$, et al. Laparoscopic splenectomy: ligasure or clip ligation? Surg Laparosc Endosc Percutan Tech 2012;22:136-8. [CrossRef]

13. Harold KL, Pollinger $H$, Matthews BD, Kercher KW, Sing RF, Heniford BT. Comparison of ultrasonic energy, bipolar thermal energy, and vascular clips for the hemostasis of small-, medium-, and large-sized arteries. Surg Endosc 2003;17:1228-30. [CrossRef]

14. Romano F, Gelmini R, Caprotti R, Andreotti A, Guaglio M, Franzoni $C$, et al. Laparoscopic splenectomy: ligasure versus EndoGIA: a comparative study. J Laparoendosc Adv Surg Tech A 2007;17:763-7. [CrossRef]

15. Miles WF, Greig JD, Wilson RG, Nixon SJ. Technique of laparoscopic splenectomy with a powered vascular linear stapler. Br J Surg 1996;83:1212-4. [CrossRef]

16. Uranüs S. Laparoscopic Partial and Total Splenectomy in non-trauma Patients. Laparosc Endosc Surg Sci 2017;24:3845.

17. Vecchio R, Marchese S, Swehli E, Intagliata E. Splenic hilum management during laparoscopic splenectomy. J Laparoendosc Adv Surg Tech A 2011;21:717-20. [CrossRef]

18. Patel AG, Parker JE, Wallwork B, Kau KB, Donaldson N, Rhodes MR, et al. Massive splenomegaly is associated with significant morbidity after laparoscopic splenectomy. Ann Surg 2003;238:235-40.

19. Unver Dogan N, Uysal II, Demirci S, Dogan KH, Kolcu G. Accessory spleens at autopsy. Clin Anat 2011;24:757-62. [CrossRef] 